\title{
Fabrication of high-resolution zone plates with wideband extreme-ultraviolet holography
}

\author{
Harun H. Solak, a) Christian David, and Jens Gobrecht \\ Laboratory for Micro and Nanotechnology, Paul Scherrer Institut, CH-5232 Villigen-PSI, Switzerland
}

(Received 11 May 2004; accepted 10 August 2004)

\begin{abstract}
We report an achromatic holographic method to fabricate high-resolution x-ray optics using coherent extreme-ultraviolet radiation from an undulator source. The interference pattern between two spherical beams, which are created using Fresnel zone plates, is recorded to produce a higher-resolution zone plate. Analytical and simulation results showing the formation of the zone plate pattern was confirmed experimentally with the production and testing of a lens with 60-nm outermost zone width. The combination of extreme-ultraviolet light, which exposes photoresists with practically no proximity effect, and holography, which guarantees the accurate placement of zones, addresses the main difficulties faced in the improvement of the resolution of x-ray lenses. Holography with extreme-ultraviolet light has the potential to produce lenses with sub-10-nm resolution. () 2004 American Institute of Physics. [DOI: 10.1063/1.1803937]
\end{abstract}

The use of $\mathrm{x}$ rays in imaging applications, such as microscopy or lithography, has long been pursued for its highresolution potential. Recent advances in the fabrication of $\mathrm{x}$-ray optics have led to unprecedented progress with the use of reflective, ${ }^{1}$ refractive, ${ }^{2}$ or diffractive optics. ${ }^{3}$ The highest resolution in $\mathrm{x}$-ray imaging is currently achieved with electron-beam-written Fresnel zone plates (FZP). These lenses are used in $\mathrm{x}$-ray imaging applications that demand high spatial resolution, such as the imaging of intracellular structures ${ }^{4}$ and magnetic domains. ${ }^{5}$ New areas of use for these optics have been emerging in recent years, such as phase-contrast x-ray microscopy, ${ }^{7}$ and advanced lithography applications. ${ }^{7,8}$ Driving the spatial resolution down to $10 \mathrm{~nm}$ and below would make a qualitative difference in the performance of x-ray imaging for all of these areas and would add new applications to the list which have length scales that are beyond the resolution capabilities of currently available optics.

In its most common form, a FZP consists of absorbing or phase shifting concentric circular rings positioned on a transparent substrate. The resolution obtained with a FZP is determined by the width of the smallest zones. Electron-beam lithography (EBL) has been used to manufacture zone plates with structures as small as $20 \mathrm{~nm},{ }^{3}$ which provide optical resolution performance in the same order. ${ }^{9}$ The width of the smallest zones that can be manufactured with EBL is mainly limited by the proximity effect, due to the long range of secondary electrons. ${ }^{10}$ Another difficulty in this lithographic method concerns the placement of zones with an accuracy better than the zone width, which is required for achieving diffraction limited optical performance. Interferometer controlled stages are used to address this problem, ${ }^{11}$ but residual errors remain because a direct measurement of the beam position on the substrate is not provided.

FZP lenses have also been made with laser holography, ${ }^{12}$ which is a powerful technique to produce diffractive optics with excellent control of the phase. ${ }^{13}$ Yun et al. proposed using soft $\mathrm{x}$ rays to achieve higher resolution in holographic fabrication. ${ }^{14}$ They described a spatial-frequency multiplica-

\footnotetext{
${ }^{a)}$ Electronic mail: harun.solak@psi.ch
}

tion scheme, where interference between two different diffraction orders of a "parent" zone plate is used to create a higher-resolution "daughter." In this method, the illuminating beam has to be spatially coherent and its spectral width given by $\Delta \lambda / \lambda$ (where $\Delta \lambda$ is the full width at half maximum of the spectrum) has to be smaller than the inverse of the number of zones of the parent zone plate in order to achieve the desired interference. These conditions make the technique effectively impractical, since the currently available soft x-ray sources cannot provide the necessary photon flux satisfying both the spatial coherence and the spectral-width requirements. We have recently developed an achromatic interference method using soft $\mathrm{x}$ rays to make nanometer scale linear periodic patterns, where beams from a number of diffraction gratings positioned on a transparent mask interfere to form a periodic intensity pattern. ${ }^{15}$ This spatial-frequency multiplication technique can be used with wideband light if the diffraction gratings on the mask have a single and constant period, ensuring that all interfering beams reaching the image plane travel the same optical path length. The illumination requirements of this scheme are satisfied by undulators in the soft $\mathrm{x}$ ray and extreme-ultraviolet (EUV) region, which emit radiation with good spatial coherence and a rather wide bandwidth $(\Delta \lambda / \lambda \approx 1 \%-5 \%) .{ }^{16}$

The achromatic spatial-frequency multiplication method discussed above is not directly applicable to the production of zone plates, because of the dependence of the zone period on the radius in a FZP. In the following, we will show that this limitation can be overcome with the holographic scheme illustrated in Fig. 1, where beams interfering at a certain image plane travel equal optical paths. Assuming that the mask in Fig. 1 is illuminated with a unity amplitude plane wave at normal incidence, we can write the amplitudes of the two interfering spherical beams in the Fresnel approximation as

$$
U_{n}=\frac{A_{n} \exp (i k z) f_{n}}{i \lambda\left(f_{n}+z\right)} \exp \left(-i \frac{k r^{2}}{2} \frac{1}{f_{n}+z}\right), \quad n=1,2,
$$

where $k$ is the wave number, $A_{n}$ is the diffraction efficiency, and $f_{n}$ is the focal length of the $n^{\text {th }}$ zone plate. ${ }^{14}$ The intensity of the total field is found to be 


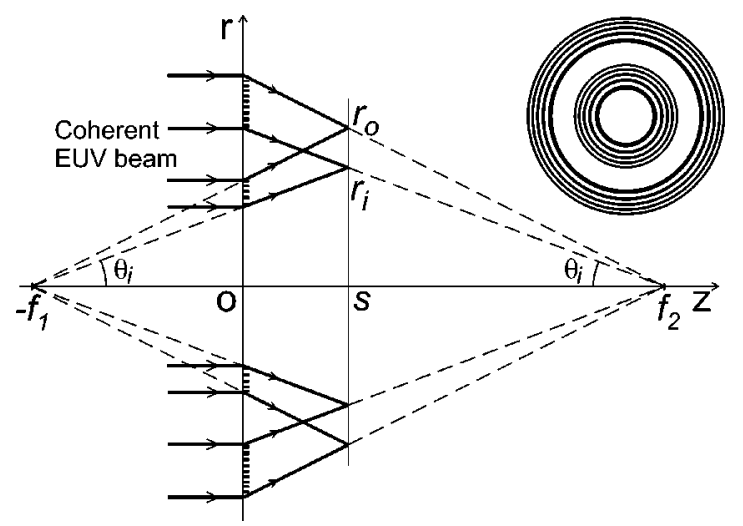

FIG. 1. The principle of achromatic holography for making a FZP. A mask containing two zone plates with focal lengths $f_{1}$ and $f_{2}$ is illuminated with spatially coherent EUV light from the left-hand side. At the image plane $(z=s)$ the diverging and converging diffraction orders from the two zone plates intersect at equal angles to form a zone plate pattern with half the zone width of the parent zone plates. The inner and outer radii of the parent zone plates can be chosen so that in a certain annular range at the image plane, the undesired diffraction orders are absent. A schematic frontal view of the mask is shown in the upper right-hand side.

$$
\begin{aligned}
I= & \left(\frac{A_{1} f_{1}}{\lambda\left(f_{1}+z\right)}\right)^{2}+\left(\frac{A_{2} f_{2}}{\lambda\left(f_{2}-z\right)}\right)^{2} \\
& +\frac{2 A_{1} A_{2} f_{1} f_{2}}{\lambda^{2}\left(f_{1}+z\right)\left(f_{2}-z\right)} \cos \left[\frac{k r^{2}}{2}\left(\frac{1}{f_{1}+z}+\frac{1}{f_{2}-z}\right)\right] .
\end{aligned}
$$

At the image plane, $z=s$, this equation simplifies to give

$$
I=\left(\frac{A_{1} f_{1}}{\lambda D}\right)^{2}+\left(\frac{A_{2} f_{2}}{\lambda D}\right)^{2}+\frac{2 A_{1} A_{2} f_{1} f_{2}}{\lambda^{2} D^{2}} \cos \left(\frac{k r^{2}}{D}\right),
$$

where $s=\left(f_{2}-f_{1}\right) / 2$, and $D=\left(f_{1}+f_{2}\right) / 2$. The cosine term in the above expression is periodic in $r^{2}$, and has the correct form for a zone plate with a focal length of $f=D / 2$. After some straightforward manipulation and using $D / 4$ as a typical value for the distance $s$ between the mask and the image plane, we can show that the interference pattern formed by the two beams is invariant with respect to the wavelength within the spectral region given by

$$
\frac{\Delta \lambda}{\lambda_{0}} \ll \frac{4}{\sqrt{N}},
$$

where $N$ is the maximum zone number of the daughter zone plate. This shows the significant improvement in the bandwidth of usable radiation, since without the achromatic arrangement the allowed bandwidth is of the order of $1 / N$. Using similar arguments we can show that the depth of focus, where the image is formed, is given by

$$
\Delta z \ll \frac{l_{c}}{N A^{2}},
$$

where $N A$ is the numerical aperture of the holographic set up and $l_{c}=\lambda_{0}^{2} / \Delta \lambda$ is the longitudinal coherence length of the light.

Numerical simulations based on the method of stationary phases $^{14}$ confirmed the formation of fringes with strong modulation in the image plane with wideband illumination (Fig. 2). When the illumination is perfectly monochromatic, the fringes formed by the two interfering beams have good

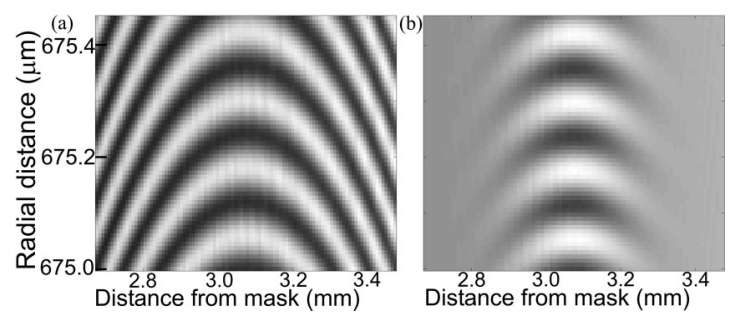

FIG. 2. Intensity distribution calculated with the method of stationary phases near the image plane for (a) monochromatic, and (b) wide spectrum $(\Delta \lambda / \lambda=0.04)$ illumination. The fringes are part of the elliptical curves corresponding to the constant optical path difference surfaces between the two sources located at the respective foci of the two parent zone plates. In this calculation we assumed the duty cycles of the two parent zone plates, and hence their diffraction efficiencies to be the same. The focal lengths and the dimensions of the parent zone plates were the same as the ones used in the experiment.

visibility everywhere, whereas in the wideband case they remain visible only in a certain range around the image plane as predicted by Eq. (5).

We confirmed the applicability of the proposed method experimentally by making condenser lenses for phasecontrast x-ray microscopy which requires large-diameter, ring-shaped zone plates with high-resolution structures. ${ }^{17}$ The holographic masks were written with EBL on thin silicon-nitride membranes coated with a thin film of chromium. The patterns, written in an electron-beam resist, were later transferred into the underlying $\mathrm{Cr}$ and $\mathrm{Si}_{3} \mathrm{~N}_{4}$ layers with reactive ion etching. ${ }^{18}$ The inner and outer zone plates on the mask had the smallest zone widths of about $120 \mathrm{~nm}$ and focal lengths of 9.216 and $15.365 \mathrm{~mm}$ at $13.4-\mathrm{nm}$ wavelength, respectively. The annular ranges of these two zone plates were chosen to create a daughter zone plate in the annular range of $0.580-0.690 \mathrm{~mm}$.

The masks made with EBL were used in holographic exposures at the X-ray interference lithography (XIL) beamline of the Swiss Light Source. The beamline, built as a branch on a high-resolution spectroscopy beamline, ${ }^{19}$ provides spatially filtered coherent illumination in the EUV region. By using either the diffracted or the reflected beam from the beamline monochromator grating, it is possible to obtain either a highly monochromatic or a wideband beam, respectively. For the holographic exposures, the undulator source was set to a central wavelength of $13.4 \mathrm{~nm}$ and a spectral width of $\Delta \lambda / \lambda=0.04$. Test exposures confirmed the large depth of focus of more than $100 \mu \mathrm{m}$ predicted by the results of our analytical derivations and simulations. The substrates and the pattern transfer scheme for the holographically exposed zone plates were the same as those used for the EBL-made masks. Scanning electron microscopy (SEM) examination of the resulting lenses showed zones formed within the designed annular region with the predicted zone width of $60 \mathrm{~nm}$, i.e., two times smaller than the EBL-written structure width in the mask (Fig. 3).

The performance of the condenser lenses made with EUV holography was measured at the same XIL beamline. A pinhole was used to illuminate the FZP under test with coherent $x$ rays. The intensity profile of the image formed by the lens was measured with a knife edge scan, which showed that this large lens of about 1.4-mm diam focused the light to a $0.77-\mu \mathrm{m}$-diam spot as measured between the $20 \%-80 \%$ points of the scan (Fig. 4). We attribute the difference between the achieved spot size and the diffraction-limited size 


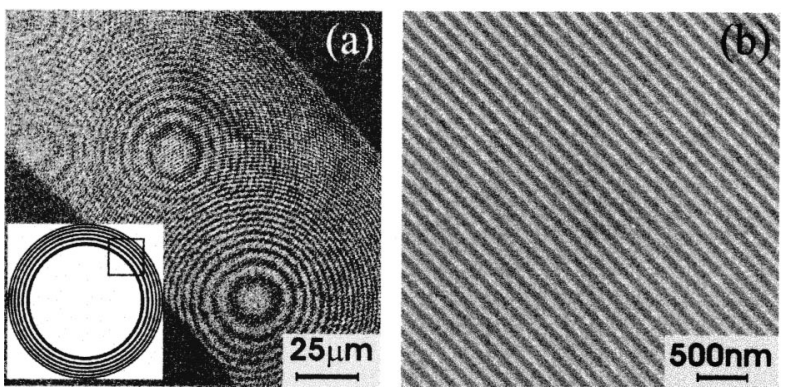

FIG. 3. SEM images of the holographically made FZP after the recorded pattern was etched into the $\mathrm{Cr}$ film and the $\mathrm{Si}_{3} \mathrm{~N}_{4}$ support membrane. (a) Low-resolution image showing Moiré fringes in the annular range where the zone structures were formed. The Moiré fringes are formed between the SEM scan lines and the circular zone structures. The schematic inset indicates the area of the ring-shaped FZP where the SEM image was acquired. (b) Higher-resolution image showing etched zones with about 66-nm zone width. The image was taken approximately in the middle of the annular range shown in (a).

of $0.20 \mu \mathrm{m}$ to the zone placement errors in the EBL-written parent zone plates. In fact, we measured these errors to be more than $300 \mathrm{~nm}$ (i.e., much larger than the zone width) with a SEM equipped with an interferometer controlled stage. The achieved performance is satisfactory for the function of the condenser lens, which is to illuminate samples with a high numerical aperture beam rather than to produce a spot size comparable to the outer zone width of the lens. Maintaining the zone placement accuracy is much easier with smaller optics (microzone plates) typically used in highresolution x-ray imaging. ${ }^{9}$ This experiment confirms our theoretical predictions and demonstrates the practicality of the approach with an available EUV source and beamline.

The scheme depicted in Fig. 1 can be used to make ring-shaped FZP with an obstructed region in the center covering more than $50 \%$ of the radial range. The condenser lens reported in this Letter required a large obstructed region

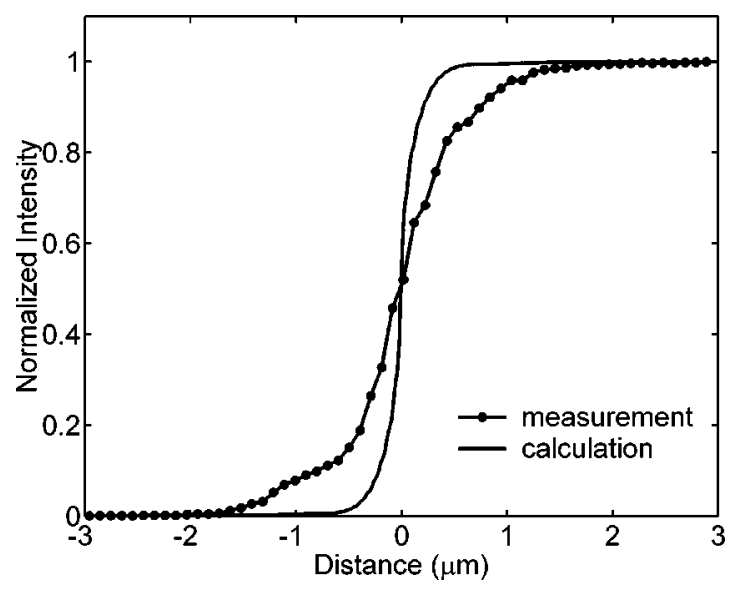

FIG. 4. Theoretically calculated and measured knife edge scans across the focused beam. A lithographically made open slit measuring $20 \times 100 \mu \mathrm{m}^{2}$ in a thin, but opaque, membrane was used for this purpose. A photodiode behind the slit was used to measure the transmitted light intensity. The measured data was normalized but no background was removed. The calculated curve includes the broadening effect of the illuminating spectrum. For this test the monochromator in the beamline was set to provide a spectral bandwidth of $\Delta \lambda / \lambda=3 \times 10^{-4}$.
( $84 \%$ of the lens radius), which is responsible for the reduction in the predicted resolution down to $0.2 \mu \mathrm{m}$ from a value close to the outermost zone width of $60 \mathrm{~nm}$. This impact on resolution, which is much less severe for achievable, smaller obstruction ratios, has to be taken into account in the design and use of FZP made with this technique.

When used with state of the art EBL-made zone plates, spatial-frequency multiplication should readily lead to zone plates with $10-\mathrm{nm}$ resolution. ${ }^{3,9}$ The ultimate limit in the holographic fabrication is equal to one-quarter of the wavelength used, which corresponds to about $3.5 \mathrm{~nm}$ for a 13.4-nm wavelength. The use of laser holography for making the parent zone plates can free the process from the artifacts of EBL. The achromatic scheme makes it possible to utilize the full bandwidth of the wideband source to perform the exposures in practical times. As zone-plate-based x-ray microscopy matures into a commercialization phase, this method offers a high throughput means for fabrication of the necessary optics with exposures taking seconds, instead of hours, required for EBL. Other x-ray optical elements demanding high spatial resolution, such as variable period spectrometric gratings and linear zone plates, can be made with the described achromatic EUV holography.

The authors thank J. F. van der Veen, L. Heyderman, and B. Nöhammer for discussions. Part of this work was performed at the Swiss Light Source, Paul Scherrer Institute, Villigen, Switzerland.

${ }^{1}$ O. Hignette, P. Cloetens, W.-K. Lee, W. Ludwig, and G. Rostaing, J. Phys. IV 104, 231 (2003).

${ }^{2}$ A. Snigirev, V. Kohn, I. Snigireva, and B. Lengeler, Nature (London) 384, 49 (1996).

${ }^{3}$ M. Peuker, Appl. Phys. Lett. 78, 2208 (2001).

${ }^{4}$ C. A. Larabell and M. A. Le Gros, Mol. Biol. Cell 15, 957 (2003).

${ }^{5}$ P. G. Evans, E. D. Isaacs, G. Aeppli, Z. Cai, and B. Lai, Science 295, 1042 (2002).

${ }^{6}$ E. Di Fabrizio, D. Cojoc, S. Cabrini, B. Kaulich, J. Susini, P. Facci, and T. Wilhein, Opt. Express 11, 2278 (2003).

${ }^{7}$ H. I. Smith, J. Vac. Sci. Technol. B 14, 4318 (1996).

${ }^{8}$ Y. Wang, W. Yun, and C. Jacobsen, Nature (London) 424, 50 (2003).

${ }^{9}$ W. L. Chao, E. Anderson, G. P. Denbeaux, B. Harteneck, J. A. Liddle, D. L. Olynick, A. L. Pearson, F. Salmassi, C. Y. Song, and D. T. Attwood, Opt. Lett. 28, 2019 (2003).

${ }^{10}$ T. H. P. Chang, J. Vac. Sci. Technol. 12, 1271 (1975).

${ }^{11}$ E. H. Anderson, D. L. Olynick, B. Harteneck, E. Veklerov, G. Denbeaux, W. L. Chao, A. Lucero, L. Johnson, and D. H. Attwood, J. Vac. Sci. Technol. B 18, 2970 (2000).

${ }^{12}$ G. Schmahl, D. Rudolph, and P. Guttmann, in X-ray Microscopy, edited by G. Schmahl and D. Rudolph (Springer, Heidelberg, 1984), pp. 63-74.

${ }^{13}$ P. T. Konkola, C. G. Chen, R. K. Heilmann, C. M. Joo, J. C. Montoya, C. H. Chang, and M. L. Schattenburg, J. Vac. Sci. Technol. B 21, 3097 (2003).

${ }^{14}$ W. B. Yun and M. R. Howells, J. Opt. Soc. Am. A 4, 34 (1987).

${ }^{15}$ H. H. Solak, C. David, J. Gobrecht, L. Wang, and F. Cerrina, J. Vac. Sci. Technol. B 20, 2844 (2002).

${ }^{16}$ D. Attwood, K. Halbach, and K. J. Kim, Science 228, 1265 (1985).

${ }^{17}$ U. Neuhäusler, G. Schneider, W. Ludwig, and D. Hambach, J. Phys. IV 104, 567 (2003).

${ }^{18}$ H. H. Solak, C. David, J. Gobrecht, V. Golovkina, F. Cerrina, S. O. Kim, and P. F. Nealey, Microelectron. Eng. 67-68, 56 (2003).

${ }^{19}$ U. Flechsig, L. Patthey, and C. Quitmann, Nucl. Instrum. Methods Phys. Res. A 467, 479 (2001). 